\title{
Parameter identification in multinomial processing tree models
}

\author{
Verena D. Schmittmann, Conor V. Dolan, and Maartje E. J. RaiJmakers \\ University of Amsterdam, Amsterdam, The Netherlands
}

AND

WiLLIAM H. BATCHELDER

University of California, Irvine, California

\begin{abstract}
Multinomial processing tree models form a popular class of statistical models for categorical data that have applications in various areas of psychological research. As in all statistical models, establishing which parameters are identified is necessary for model inference and selection on the basis of the likelihood function, and for the interpretation of the results. The required calculations to establish global identification can become intractable in complex models. We show how to establish local identification in multinomial processing tree models, based on formal methods independently proposed by Catchpole and Morgan (1997) and by Bekker, Merckens, and Wansbeek (1994). This approach is illustrated with multinomial processing tree models for the source-monitoring paradigm in memory research.
\end{abstract}

Multinomial processing tree (MPT) models form an increasingly popular class of stochastic models for categorical data that have applications in a variety of research areas in cognitive, differential, and social psychology (e.g., Batchelder \& Riefer, 1999, 2007; Erdfelder et al., 2009; Stahl, 2006). For example, Batchelder and Riefer (1999) discussed over 80 applications of MPT models in various areas of psychology, including memory, perception, and reasoning. The statistical theory of MPT models, including maximum likelihood (ML) parameter estimation, overall model testing, and tests of specific hypotheses within models, has been discussed by Hu and Batchelder (1994) and by Riefer and Batchelder (1988). Flexible software to fit MPT models by means of ML estimation has been developed by $\mathrm{Hu}$ and Phillips (1999), Moshagen (2010), Rothkegel (1999), and Stahl and Klauer (2007). MPT models entail a reparameterization of the cell probabilities of the multinomial or product-multinomial distribution (Andersen, 1980; Bishop, Fienberg, \& Holland, 1975) in terms of parameters assumed to represent the probabilities of underlying cognitive processes. The underlying cognitive architecture is represented as a rooted tree, in which each branch represents a processing sequence leading to an observable categorical response. More than one branch may terminate in a given response.

In MPT models, as in all statistical models, global model identification (the ability to infer unique parameter values from data) is an important issue. A common cause of nonidentifiability of a model is parameter redundancy, in which case the likelihood of the model can be expressed as a function of fewer than the original number of parameters (Catchpole \& Morgan, 1997). If a model is not parameter redundant, it is locally, and possibly even globally, identified; we go into the details of local and global identification in terms of MPT models below.

In an MPT model, there is a function $\boldsymbol{f}(\boldsymbol{\theta})$ that maps the model's parameter space into the set of possible multinomial probability distributions. Global identification can be established by proving that inequality of two parameter vectors $\boldsymbol{\theta} \neq \boldsymbol{\theta}^{*}$ implies inequality of the modeled multinomial cell probability vectors $\boldsymbol{f}(\boldsymbol{\theta}) \neq \boldsymbol{f}\left(\boldsymbol{\theta}^{*}\right)$ for all parameter vectors $\boldsymbol{\theta}$ and $\boldsymbol{\theta}^{*}$ in the parameter space (Hu \& Batchelder, 1994; Meiser, 2005; Riefer \& Batchelder, 1988; see also Bishop et al., 1975, p. 510). The required calculations for establishing global identification can become tedious, and even intractable, in complex models. In this case, the next best thing is to establish local identification; that is, $\boldsymbol{\theta} \neq \boldsymbol{\theta}^{*}$ implies $\boldsymbol{f}(\boldsymbol{\theta}) \neq \boldsymbol{f}\left(\boldsymbol{\theta}^{*}\right)$ for all $\boldsymbol{\theta}$ in an open neighborhood around $\theta^{*}$. One can empirically investigate local identification at a specific point $\boldsymbol{\theta}_{G}$ in the parameter space by fitting the generating MPT model to artificial (simulated) data generated by $\boldsymbol{\theta}_{G}$, or to exact summary statistics - that is, the expected cell counts, derived from the probabilities $\boldsymbol{f}\left(\boldsymbol{\theta}_{G}\right)$ implied by the generating MPT model. Obtaining parameter estimates sufficiently close to the true values $\boldsymbol{\theta}_{G}$ (in the case of simulated data), or recovering exactly the true parameter values (in the case of exact summary statistics), is a necessary condition for local identification. In addition, following such analyses, one may numerically inspect the rank of the information

V. D. Schmittmann, v.d.schmittmann@uva.nl 
matrix (i.e., the matrix of second order partial derivatives of the log of the likelihood vector function with respect to the parameter vector; Azzalini, 1996) to establish that it is positive definite (Viallefont, Lebreton, Reboulet, \& Gory, 1998). Various methods are available to calculate the information matrix (e.g., Dolan \& Molenaar, 1991; Moshagen, 2010; Stahl \& Klauer, 2007; Viallefont et al., 1998). However, as we shall see below, the expression for the expected information matrix is quite simple in parameterized multinomial models.

An alternative approach to establish local identification is by means of symbolic calculation. This approach has the advantage that it does not require an arbitrary choice of parameter values and avoids numerical computations (e.g., minimization) altogether. It does require symbolic calculations, but these can be carried out readily using computer algebra software. Catchpole and colleagues (e.g., Catchpole \& Morgan, 1997, 2001; Catchpole, Morgan, \& Freeman, 1998; Catchpole, Morgan, \& Viallefont, 2002) developed this method in a series of articles for capture-recapture models. They also considered the problem of deriving identifying constraints, given an unidentified model. Independently, Bekker, Merckens, and Wansbeek (1994) developed this method in the context of structural equation modeling. The aim of this article is to discuss this method in the context of MPT modeling, and to present several illustrations using the open-source program Maxima (Dodier, 2009; we used version wxMaxima 0.8.4). Input code for the commercial program Maple (Monagan et al., 2008) is available upon request.

\section{MPT Models}

MPT models specify a reparameterization of the category probabilities of the multinomial or productmultinomial distribution (Andersen, 1980; Bishop et al., 1975) in terms of parameters derived from a substantive theory. Assume a random sample of $N$ independent and identically distributed observations, each of which can be classified into one of $J$ discrete, mutually exclusive, and jointly exhaustive response categories, $C_{1}, C_{2}, \ldots, C_{J}$. Let $n_{j}$ be the number of observations in $C_{j}$, and let $\boldsymbol{D}=$ $\left(n_{1}, n_{2}, \ldots, n_{j}, \ldots, n_{J}\right)$ be the data vector of counts. The joint distribution of the data $\boldsymbol{D}$ is given by the multinomial distribution

$$
P\left(\boldsymbol{D} ; p_{1}, \ldots, p_{J}\right)=N ! \prod_{j=1}^{J} p_{j}^{n_{j}} / n_{j} !,
$$

where $p_{j}$ is the probability that an observation falls into category $C_{j}$, and $N=\sum_{j=1}^{J} n_{j}$. The set of all possible $J$-dimensional probability distributions over $J$ categories is given by $\Omega_{J}=\left\{\boldsymbol{p}: p_{j}>0, \Sigma_{j=1}^{J} p_{j}=1\right\}$. The true, model-implied category probabilities are required to lie in the interior of $\Omega_{J}$ and not on its boundary. The vector of observed data proportions $\boldsymbol{P}=\boldsymbol{D} / N$ is a point in $\Omega_{J}$.

Based on the experimental design, for example, in the case of multiple within- and/or between-participant conditions, the observations may be separated into $K$ disjoint data sets $\boldsymbol{D}_{k}$, where data set $\boldsymbol{D}_{k}$ consists of $N_{k}$ independent and identically distributed observations, which fall into one of $J_{k}$ categories $C_{k 1}, C_{k 2}, \ldots, C_{k J_{k}}$. In this case, assuming independence between data sets, the joint probability of the $K$ random vectors $\boldsymbol{D}_{k}=\left(n_{k 1}, n_{k 2}, \ldots, n_{k J_{k}}\right)$ of category counts is given by the product-multinomial distribution

$$
\begin{gathered}
P\left(\boldsymbol{D}_{1}, \ldots, \boldsymbol{D}_{K} ; p_{11}, \ldots, p_{1 J}, \ldots, p_{K 1}, \ldots, p_{K J}\right)= \\
\prod_{k=1}^{K} N_{k} ! \prod_{j=1}^{J_{k}} p_{k j}^{n_{k j}} / n_{k j} !,
\end{gathered}
$$

where $p_{k j}$ is the probability that an observation falls into category $j$ of condition $k$, and $\sum_{j=1}^{J_{k}} p_{k j}=1$, and where $N_{k}=\sum_{j=1}^{J_{k}} n_{k j}$. The set of all possible combinations of the $K$ probability vectors $\Omega_{K J}$ equals the Cartesian product of the sets of all $J_{k}$-dimensional probability vectors $\Omega_{J_{k}}$ of all conditions.

In MPT models, the product-multinomial distribution in Equation 2 is reparameterized through a vector $\boldsymbol{\theta}$ of $S$ functionally independent model parameters, $\boldsymbol{\theta}=\left(\theta_{1}, \ldots\right.$, $\left.\theta_{S}\right), 1 \leq S \leq\left(\sum_{k=1}^{K} J_{k}\right)-K$, with each $\theta_{s}$ lying within the open interval $(0,1)$ or a subinterval thereof. A vector function $\boldsymbol{f}(\boldsymbol{\theta})$ of the parameters maps each point $\boldsymbol{\theta}$ in the parameter space $\Theta$ onto a point in the space of all sets of possible probability vectors $\Omega_{K J}$, such that the productmultinomial cell probabilities $p_{k j}$ are modeled by $f_{k j}(\boldsymbol{\theta})$. Each MPT model parameter $\theta_{s}$ represents the probability of a latent cognitive event that may be involved in the generation of a manifest behavioral response. The likelihood function for the $k$ th data set is given by

$$
L_{k}\left(\boldsymbol{D}_{k} ; \boldsymbol{\theta}\right)=N_{k} ! \prod_{j=1}^{J_{k}}\left[f_{k j}(\boldsymbol{\theta})\right]^{n_{k j}} / n_{k j} !
$$

The likelihood function for the joint or productmultinomial model is the product of the $K$ likelihood functions:

$$
L\left(\boldsymbol{D}_{1}, \ldots, \boldsymbol{D}_{K} ; \boldsymbol{\theta}\right)=\prod_{k=1}^{K} L_{k}\left(\boldsymbol{D}_{k} ; \boldsymbol{\theta}\right) .
$$

\section{Identification}

Subject to various regularity conditions (Bishop et al., 1975, p. 510; Read \& Cressie, 1988), maximization of the expression in Equation 4 produces unique ML parameter estimates of $\boldsymbol{\theta}, \boldsymbol{\theta}_{\mathrm{ML}}$, which converge to the true parameter vector $\boldsymbol{\theta}_{\mathrm{T}}$ as the number of observations, $N$, is increased. The regularity conditions, which ensure that such unique ML estimates actually exist, stipulate inter alia that $\boldsymbol{\theta} \neq$ $\boldsymbol{\theta}_{\mathrm{T}}$ implies $\boldsymbol{f}(\boldsymbol{\theta}) \neq \boldsymbol{f}\left(\boldsymbol{\theta}_{\mathrm{T}}\right)$, which is satisfied if $\boldsymbol{\theta} \neq \boldsymbol{\theta}^{*}$ implies $\boldsymbol{f}(\boldsymbol{\theta}) \neq \boldsymbol{f}\left(\boldsymbol{\theta}^{*}\right)$ - that is, the global identifying condition considered in the context of MPT modeling by $\mathrm{Hu}$ and Batchelder (1994), Meiser (2005), and Riefer and Batchelder (1988). But demonstrating that this condition is satisfied can be difficult in complex models, even using computer algebra programs such as Maple or Maxima. We may, therefore, have to resort to other methods. As mentioned above, Viallefont et al. (1998) suggested cal- 
culating the rank of the observed information matrix at the point $\boldsymbol{\theta}_{\mathrm{ML}}$ as an empirical (numerical) test of local identification. They show that the rank of this information matrix equals the number of identified parameters. This is also the standard method of establishing empirical (local) identification in structural equation modeling software, such as LISREL (Bollen, 1989; Jöreskog \& Sörbom, 1989). However, numerical tests of local identification depend on arbitrarily chosen parameter values. This disadvantage can be circumvented using a symbolic approach. To establish local identification, Catchpole and colleagues (Catchpole, Kgosi, \& Morgan, 2001; Catchpole \& Morgan, 1997; Catchpole et al., 1998; Catchpole et al., 2002) and Bekker et al. (1994) suggested inspecting the rank of the $\left(\sum_{k=1}^{K} J_{k} \times S\right)$-dimensional Jacobian matrix, $\mathrm{J}(\boldsymbol{\theta})=$ $\partial \boldsymbol{f}(\boldsymbol{\theta}) / \partial \boldsymbol{\theta}$ using symbolic manipulation. A necessary condition for global identification is that this matrix has full column rank, which can be established using computer algebra programs. In the case of parameterized multinomial models, one can cast the problem in terms of the rank of the Jacobian matrix or the information matrix. Specifically, the $(S \times S)$-dimensional information matrix $\mathrm{I}(\boldsymbol{\theta})$ equals $\mathrm{J}(\boldsymbol{\theta})^{\mathrm{t}} F(\boldsymbol{\theta}) \mathrm{J}(\boldsymbol{\theta})$, where the superscript " $\mathrm{t}$ " indicates the transpose matrix and $F(\boldsymbol{\theta})$ is a diagonal matrix with the parameterized multinomial probabilities $f(\theta)$ on the diagonal (Bishop et al., 1975, p. 512). ${ }^{1}$ Regularity conditions (Bishop et al., 1975, p. 510) stipulate that the components of $\boldsymbol{f}(\boldsymbol{\theta})$ be positive (specifically $0<\boldsymbol{f}_{k j}(\theta)<1$ ), so that $F(\theta)$ is positive definite by these conditions. Consequently, the $\mathrm{I}(\boldsymbol{\theta})$ is positive definite - that is, full rank - if and only if $\mathrm{J}(\boldsymbol{\theta})$ is full column rank. Following Catchpole and colleagues (e.g., Catchpole et al., 2001; Catchpole \& Morgan, 1997; Catchpole et al., 1998; Catchpole et al., 2002), we focus on establishing the rank of $\mathrm{J}(\boldsymbol{\theta})$ in MPT models symbolically, since this is computationally more convenient than inspecting the symbolic expected information matrix. The number of parameters $S$ equals the sum of the rank of the Jacobian matrix, and of the dimension of its null space. The null space of a matrix $A$ is defined as the set of all vectors $x$ for which $A x=0$; that is, the null space spans the subspace mapped onto the null vector (see Basilevsky, 1983, pp. 119-120). In particular, the null space of the Jacobian matrix spans the direction field along which the gradient of $\boldsymbol{f}(\boldsymbol{\theta})$ is zero, and along which the likelihood surface has a flat ridge (Catchpole \& Morgan, 1997). If all parameters are locally identified, the Jacobian matrix is full rank, and the null space contains only the null vector; that is, only the null vector is mapped onto the zero vector. If the Jacobian matrix is not full rank but is rank deficient, the null space can be used to examine which of the parameters are not identified. Only those parameters that correspond to the elements that are zero in all vectors spanning the null space are identified. This use of the null space is illustrated below.

We should note that the present condition of a full rank Jacobian matrix guarantees local identification, in the sense that its fulfillment implies that the model cannot be reparameterized with fewer than $S$ parameters. For this reason, Catchpole and colleagues (Catchpole et al.,
2001; Catchpole \& Morgan, 1997; Catchpole et al., 1998; Catchpole et al., 2002) emphasized its role in detecting parameter redundancy. Catchpole and Morgan (1997) distinguished two hierarchically ordered kinds of locally identified models: essentially and conditionally full rank models. In essentially full rank models, the Jacobian matrix is full rank in the whole parameter space, whereas in conditionally full rank models there exist parameter values for which the rank of the Jacobian matrix is full; however, the model is parameter redundant, conditional on specific parameter values or constraints on the parameter space. But even an essentially full rank Jacobian matrix does not necessarily imply the global identification requirement that $\boldsymbol{\theta} \neq \boldsymbol{\theta}_{\mathrm{T}} \Rightarrow \boldsymbol{f}(\boldsymbol{\theta}) \neq \boldsymbol{f}\left(\boldsymbol{\theta}_{\mathrm{T}}\right)$. To see this, consider the following example of flipping two coins $N$ times, each with an independent and identically distributed probability of head of $q$. The possible categories are "head twice" $(\mathrm{HH})$, "different" (D), and "tail twice" (TT), and the respective probabilities are $\boldsymbol{f}(\theta)=\left[q^{2}, q(1-q),(1-q)^{2}\right]$. The subset of probabilities generated by the model is restricted to the curve shown in the left panel of Figure 1. As $q$ ranges between 0 and $1, \boldsymbol{f}(q)$ follows a one-dimensional curve in the set of all possible probability vectors. Now, suppose that the categories $\mathrm{HH}$ and TT are taken together into one category: "same." This is a four-branch, two-category binary MPT. The category probability of "same" equals $f_{\text {same }}(\theta)=q^{2}+(1-q)^{2}$, and the probability of "different" equals $f_{\text {different }}(\theta)=2(1-q) q$. The parameter vector is $\boldsymbol{\theta}=[q]$, with $0<q<1$, and the Jacobian $\mathrm{J}(\theta)$ is essentially full column rank, so there is no parameter redundancy. But the model does not satisfy $\boldsymbol{\theta} \neq \boldsymbol{\theta}_{\mathrm{T}} \Rightarrow$ $\boldsymbol{f}(\boldsymbol{\theta}) \neq \boldsymbol{f}\left(\boldsymbol{\theta}_{\mathrm{T}}\right)$, because $\boldsymbol{\theta}=[q]$ and $\boldsymbol{\theta}^{*}=[1-q]$ yield the same cell probabilities $f(\theta)=f\left(\theta^{*}\right)$, as shown in the right panel of Figure 1.

One way to solve this problem in MPT models is to impose appropriate parameter boundaries and thereby restrict the parameter space.

\section{Examples}

Below we investigate local identification, using the Jacobian rank method in two MPT models for source monitoring. Source monitoring is a highly productive paradigm in the study of memory (Batchelder \& Riefer, 1990). In a source-monitoring experiment, participants first study items that originate from two or more sources, such as distinct word lists, speakers, or perceptual modalities. The participants are then shown items that they may or may not have studied previously, and they are asked to indicate whether the items are new (i.e., distractors) or originate from one of the sources. Batchelder and Riefer (1990; see also Bayen, Murnane, \& Erdfelder, 1996; Meiser \& Bröder, 2002) developed MPT models to analyze sourcemonitoring data. These models allow one to estimate parameters, which are associated with various cognitive processes involved in source monitoring. These processes include item detection and source discrimination. Here we consider two MPT models for source monitoring: the two-high-threshold model (Bayen et al., 1996) and the low-threshold model (Batchelder, Hu, \& Riefer, 1994). 

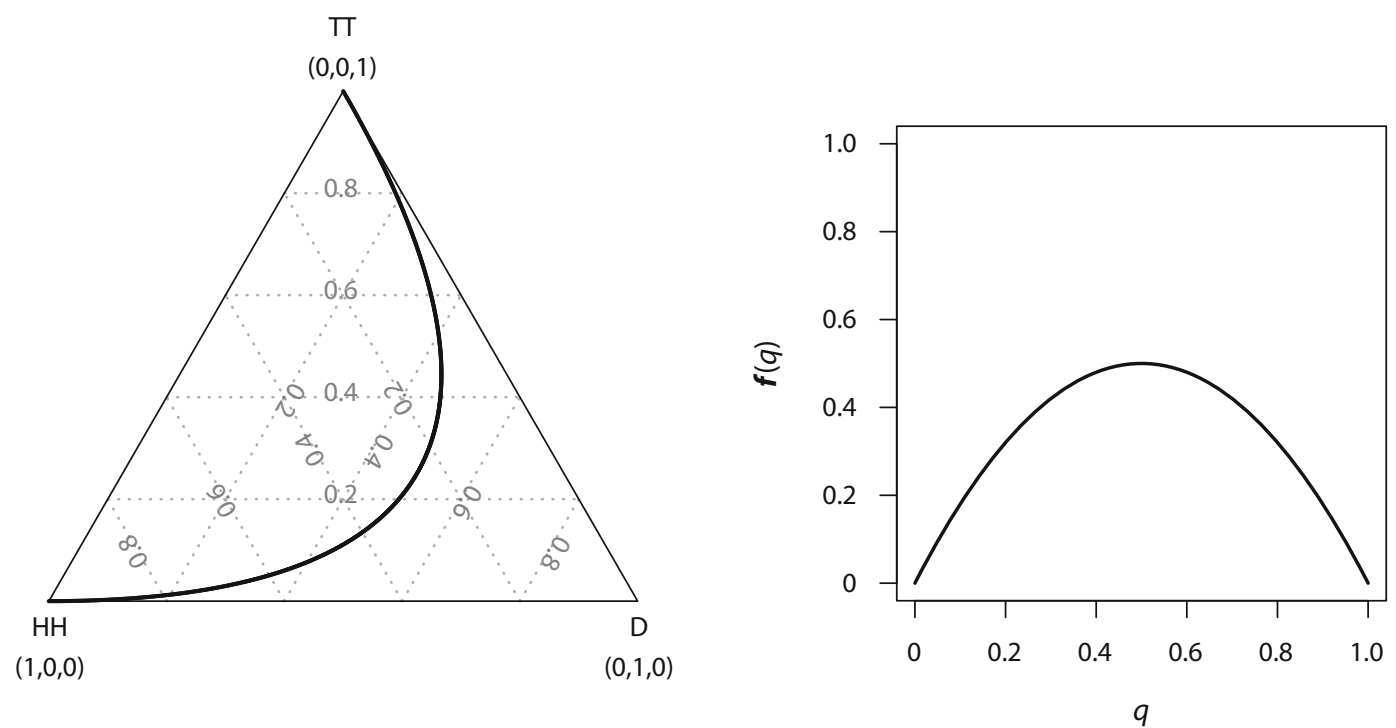

Figure 1. Left panel: Ternary plot of the probability space of the coin toss example with three categories (head twice $[\mathrm{HH}]$, different $[\mathrm{D}]$, and tail twice $[\mathrm{TT}])$. The solid curve represents the subspace of probability vectors $f(q)$ given by the model as $q$ ranges between 0 and 1. Right panel: Probability of category "same" as a function of $q$.

The models share the characteristic that a separate tree is specified for each type of item, where type is defined in terms of the item's source (e.g., Source A, Source B, Source C, or new).

Two-high-threshold model. The two-high-threshold multinomial processing tree model (2HTSM; Bayen et al., 1996) is a generalization of the one-high-threshold model (1HTSM; Batchelder \& Riefer, 1990). Bayen et al. presented an overview of the identified submodels of the 2HTSM for two sources. Keefe, Arnold, Bayen, McEvoy, and Wilson (2002) considered submodels for three sources. In the following, we examine local identification of the 2HTSM for three sources. A processing tree representation of the 2HTSM model for three sources is shown in Figure 2.

Each tree is composed of three different types of nodes: root, branch, and leaf. It starts with a root node, which is connected with the first-level branch nodes-for example, $D_{1}$ and $\left(1-D_{1}\right)$ in the tree for Source A items. The first level branch nodes are connected with the second level branch nodes - for example, $d_{1},\left(1-d_{1}\right), b$, and $(1-b)$. The third level branch nodes are connected to leaf nodes, which represent one of four category responses: A (Source A), B (Source B), C (Source C), and N (new). Note that the branch nodes with probability one-for example, between the $d_{1}$ branch node and the $A$ leaf nodeensure that all leaf nodes are on the same branch level. This facilitates the matrix representation described below.

The model consists of the following parameters. $D_{1}$ $\left(D_{2}, D_{3}\right)$ is the probability of detecting Source A (B, C) items as old; $D_{\mathrm{N}}$ is the probability of detecting new items as new; $d_{1}\left(d_{2}, d_{3}\right)$ is the probability of correctly discriminating the source of a Source A (B, C) item; $a_{1}\left(a_{2}, 1-a_{1}-a_{2}\right)$ is the probability of guessing that a detected item comes from Source A (B, C); $b$ is the probability of guessing that an undetected item is old; $g_{1}\left(g_{2}\right.$, $1-g_{1}-g_{2}$ ) is the probability of guessing that an undetected item comes from Source A (B, C), given that it was guessed as being old.

As is shown in Figure 2, this model is not parameterized as a binary MPT model, with bifurcations only. This implies restrictions on the parameter space-for example, $\left(a_{1}+a_{2}\right)<1$. However, the trifurcations can easily be reparameterized to obtain an equivalent binary MPT by replacing $a_{1}, a_{2}$, and $\left(1-a_{1}-a_{2}\right)$ with $\theta_{1}$, $\left(1-\theta_{1}\right) \theta_{2}$, and $\left(1-\theta_{1}\right)\left(1-\theta_{2}\right)$, respectively. An advantage of the binary representation is that one can resort to estimation subject solely to boundary constraints $\left(0<\theta_{1}<1\right.$; $\left.0<\theta_{2}<1\right)$. The disadvantage is that one no longer obtains parameter estimates, which are directly interpretable in terms of the cognitive process of interest-for example, $a_{2}$ versus $\left(1-\theta_{1}\right) \theta_{2}$-although these can be derived readily $\left[a_{2}=\left(1-\theta_{1}\right) \theta_{2}\right]$. The transformation of standard errors of the interpretable estimates $\left(a_{1}, a_{2}\right)$, however, is more complicated. We emphasize that these variations in parameterization have no bearing on the evaluation of local identification by inspecting the null space of the Jacobian; that is, variations in model parameterization will not render an identified model unidentified (or vice versa). In the following, the directly interpretable parameterization is used.

The following illustration parallels the Maxima input script for this model, which is shown in the Appendix. In the text, we refer between parentheses to the corresponding line numbers in the input script. The reparameterizations of the multinomial cell probabilities implied by this model may be obtained from the tree diagram by adding up all branch probabilities terminating in a specific category. Where these probabilities are already in place, these may be entered by hand (1. 44). In order to calculate these probabilities in computer algebra packages, we find the following matrix representation of an MPT convenient (11. 7-43). For 

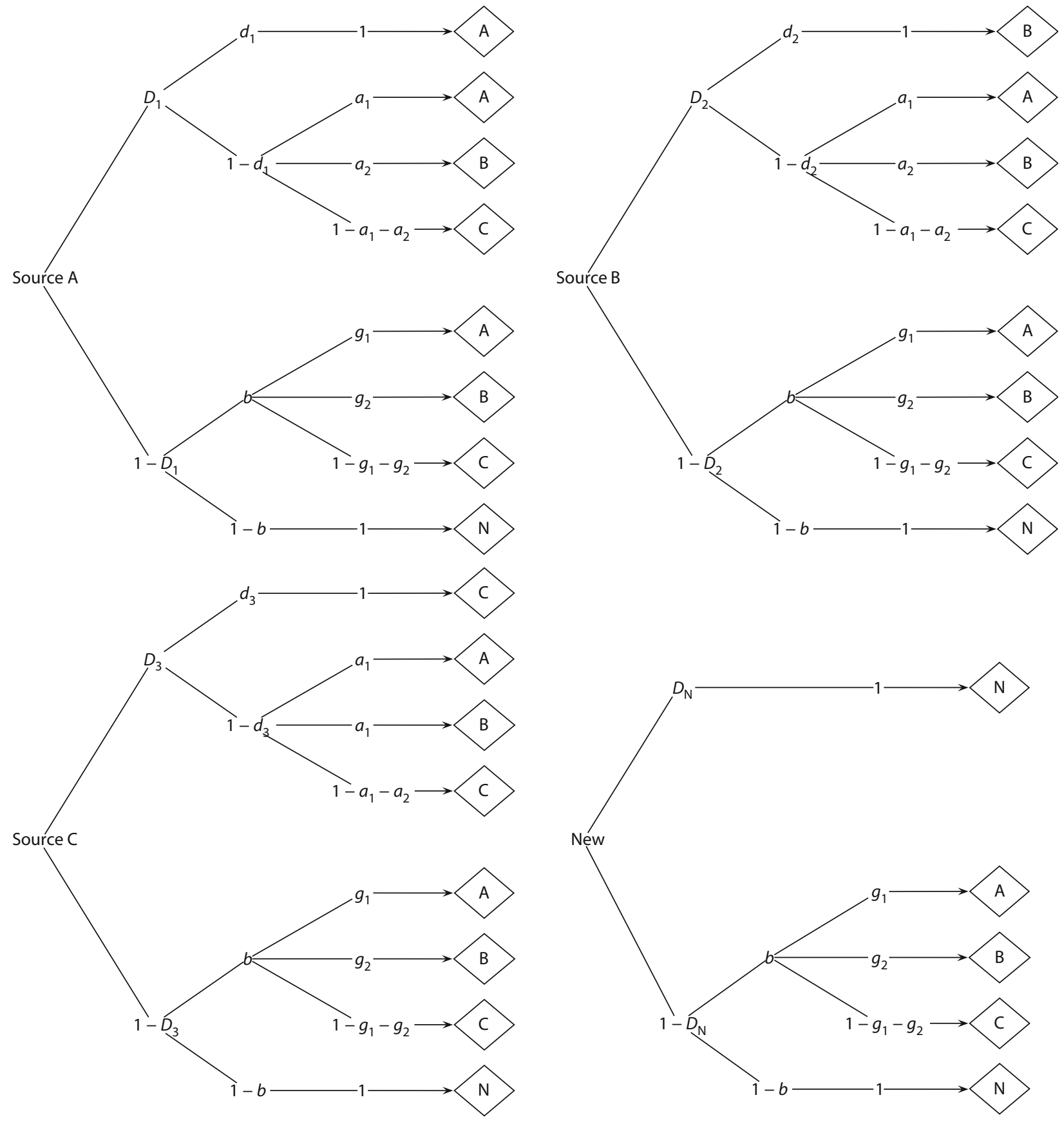

Figure 2. Two-high-threshold MPT model of source monitoring for three sources. $D_{1}\left(D_{2}, D_{3}\right)=$ probability of detecting Source $A$ $(\mathrm{B}, \mathrm{C})$ items as old; $D_{\mathrm{N}}=$ probability of detecting new items as new; $d_{1}\left(d_{2}, d_{3}\right)=$ probability of correctly discriminating the source of a Source $\mathrm{A}(\mathrm{B}, \mathrm{C})$ item; $a_{1}\left(a_{2}, 1-a_{1}-a_{2}\right)=$ probability of guessing that a detected item comes from Source $\mathrm{A}(\mathrm{B}, \mathrm{C}) ; \boldsymbol{b}=$ probability of guessing that an undetected item is old; $g_{1}\left(g_{2}, 1-g_{1}-g_{2}\right)=$ probability of guessing that an undetected item comes from Source $A$ $(B, C)$. The model includes 12 independent parameters. 
each branch level $l$ of the MPT, with $N_{\text {in }}^{l}$ incoming branches, and $N_{\text {out }}^{l}$ outgoing branches, let $M^{l}$ be a matrix of dimen$\operatorname{sion} N_{\text {out }}^{l} \times N_{\text {in }}^{l}$, where element $m_{i j}^{l}$ denotes the probability of branch $i$ given branch $j$. For instance, for Source A, the first-, second-, and third-level matrices equal

$$
M_{A}^{1}=\left(\begin{array}{c}
D_{1} \\
1-D_{1}
\end{array}\right), M_{A}^{2}=\left(\begin{array}{cc}
d_{1} & 0 \\
1-d_{1} & 0 \\
0 & b \\
0 & 1-b
\end{array}\right), \text { and }
$$

$M_{A}^{3}=\left(\begin{array}{cccc}1 & 0 & 0 & 0 \\ 0 & a_{1} & 0 & 0 \\ 0 & a_{2} & 0 & 0 \\ 0 & -a_{2}-a_{1}+1 & 0 & 0 \\ 0 & 0 & g_{1} & 0 \\ 0 & 0 & g_{2} & 0 \\ 0 & 0 & -g_{2}-g_{1}+1 & 0 \\ 0 & 0 & 0 & 1\end{array}\right)$, respectively

(11. 8-11). The last-level matrix $M_{L}$ converges the multiple branches leading to each of the $i$ response categories in the leaf nodes to an $i$-dimensional vector; for example, it maps the leaf nodes onto the four category responses. For Source A, the last-level matrix, as specified in lines 12 and 13 , equals

$$
M_{A}^{4}=\left(\begin{array}{llllllll}
1 & 1 & 0 & 0 & 1 & 0 & 0 & 0 \\
0 & 0 & 1 & 0 & 0 & 1 & 0 & 0 \\
0 & 0 & 0 & 1 & 0 & 0 & 1 & 0 \\
0 & 0 & 0 & 0 & 0 & 0 & 0 & 1
\end{array}\right) .
$$

The probability vector of an MPT model with $L$ branch levels is given by the product of matrices $M^{L} M^{L-1} \cdots M^{1}$; for example, for Source A (1. 14), the product $M_{A}$ of matrices $M_{A}^{4} M_{A}{ }^{3} M_{A}^{2} M_{A}^{1}$ is shown in Equation 5 at the bottom of the page.

The calculations are carried out analogously for the remaining three sources (11. 16-39). Consecutively, in the joint MPT model, the probability vectors of the independent trees are appended-for example, for the 2HTSM with three sources (11. 42 and 43), shown in Equation 6 at the bottom of the page.

For instance, the probability of Response A, given Source A, equals $D_{1} d_{1}+D_{1}\left(1-d_{1}\right) a_{1}+\left(1-D_{1}\right) b g_{1}$, which, given some manipulation, equals the first row in $\boldsymbol{f}(\boldsymbol{\theta})$. The vector of free parameters is $\boldsymbol{\theta}^{\mathrm{t}}=\left[D_{1}, D_{2}, D_{3}, D_{\mathrm{N}}\right.$, $\left.d_{1}, d_{2}, d_{3}, a_{1}, a_{2}, g_{1}, g_{2}, b\right]$ (1. 45). Next, with the probabil-

$$
\begin{aligned}
& M_{A}=\left(\begin{array}{c}
{\left[\left(-a_{1}+1\right) d_{1}-g_{1} b+a_{1}\right] D_{1}+g_{1} b} \\
\left(-a_{2} d_{1}-g_{2} b+a_{2}\right) D_{1}+g_{2} b \\
{\left[\left(a_{2}+a_{1}-1\right) d_{1}+\left(g_{2}+g_{1}-1\right) b-a_{2}-a_{1}+1\right] D_{1}+\left(-g_{2}-g_{1}+1\right) b} \\
(b-1) D_{1}-b+1
\end{array}\right)
\end{aligned}
$$

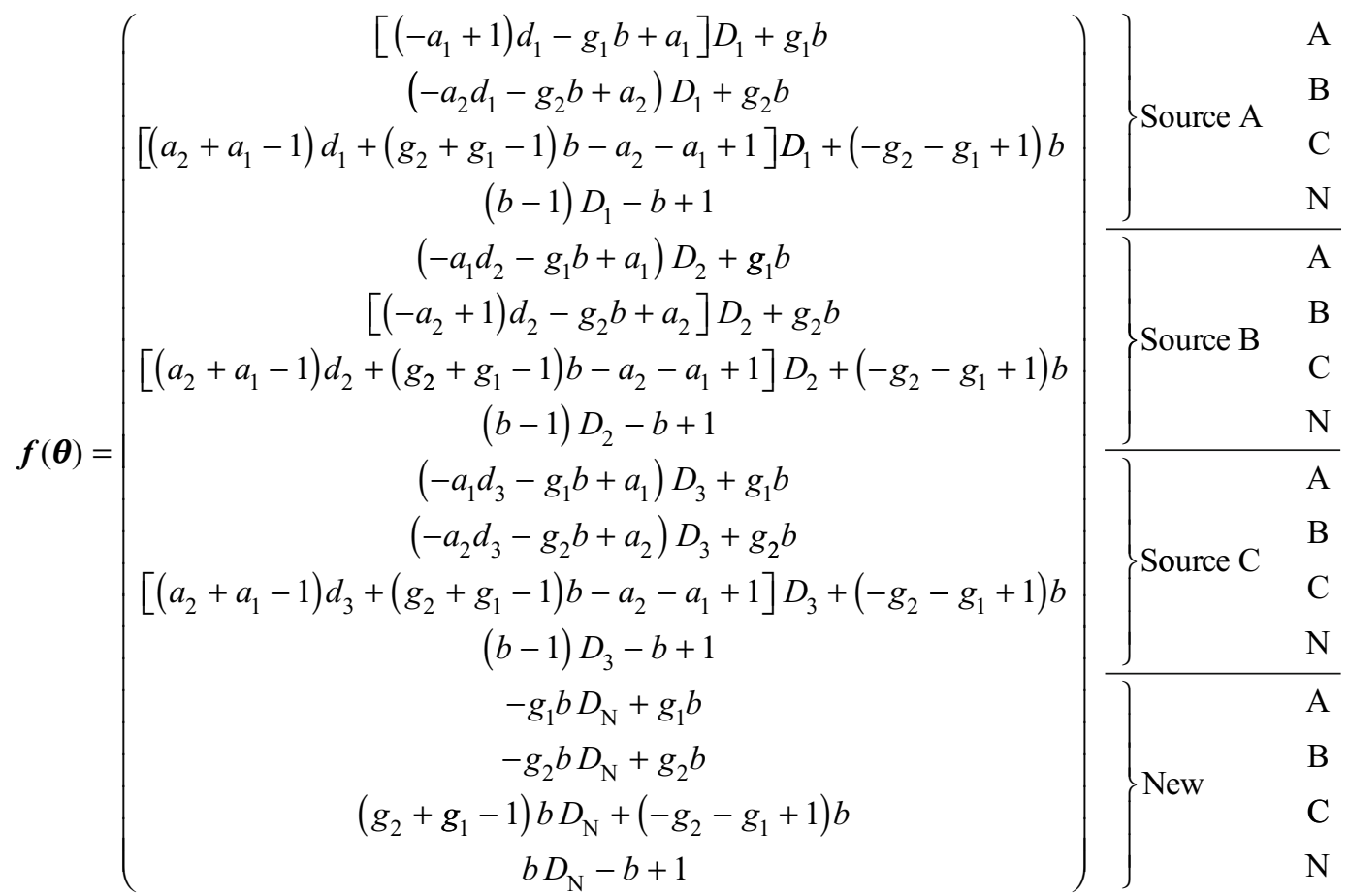


ity vector $f(\theta)$ and the vector of parameters $\theta$ in place, the Jacobian (1. 46) and its rank (1. 47) are calculated. This model is full rank - that is, locally identified - since the rank equals the number of parameters. The Maxima command nullspace $(\mathrm{J})$ returns a basis or linearly independent spanning set of the null space (1. 48). In this case, because the null space contains only the null vector itself, Maxima returns $\operatorname{span}()$. Here, only the null vector itself is projected on the null vector by the Jacobian matrix. If however, as illustrated in the next example below, the model has rank deficiency $n$, Maxima outputs $\operatorname{span}\left(v_{1}, v_{2}, \ldots, v_{n}\right)$, where the set of vectors $v_{1}, \ldots, v_{n}$ spans the null space; that is, all linear combinations of the set $v_{1}, \ldots, v_{n}$ are projected onto the zero vector.

As mentioned above, Catchpole and Morgan (1997) distinguished essentially and conditionally full rank models. In essentially full rank models, the Jacobian matrix is full rank in the whole parameter space, whereas in conditionally full rank models, the rank of the Jacobian matrix is full for some but not for all parameter values. Therefore, nested submodels of conditionally full rank models are not necessarily identified. Consider the submodel 2 HTSM $^{*}$ with equality constraints on the detection parameters $D_{1}=D_{2}=D_{3}=D$, and on the discrimination parameters $d_{1}=d_{2}=d_{3}=d$. The eight-dimensional parameter vector equals $\boldsymbol{\theta}^{\mathrm{t}}=\left[D, D_{\mathrm{N}}, d, a_{1}, a_{2}, g_{1}, g_{2}, b\right]$, and the rank of the Jacobian equals 7. Submodel 2 HTSM $^{*}$ is unidentified. The null space is calculated to determine which of the parameters are unidentified. Because the model has rank deficiency of 1, the null space is one-dimensional. Upon factorization of the resulting vector with the command factor(), Maxima outputs

$$
\operatorname{span}\left(\left(\begin{array}{c}
b^{3}\left(d^{2}-2 d+1\right)(D-1) D^{3}\left(D_{\mathrm{N}}^{2}-2 D_{\mathrm{N}}+1\right) \\
(b-1) b^{2}\left(d^{2}-2 d+1\right) D^{3}\left(D_{\mathrm{N}}^{3}-3 D_{\mathrm{N}}^{2}+3 D_{\mathrm{N}}-1\right) \\
-b^{3} d\left(d^{2}-2 d+1\right)(D-1) D^{2}\left(D_{\mathrm{N}}^{2}-2 D_{\mathrm{N}}+1\right) \\
-b^{3}(d-1)\left(g_{1}-a_{1}\right)(D-1) D^{2}\left(D_{\mathrm{N}}^{2}-2 D_{\mathrm{N}}+1\right) \\
-b^{3}(d-1)\left(g_{2}-a_{2}\right)(D-1) D^{2}\left(D_{\mathrm{N}}^{2}-2 D_{\mathrm{N}}+1\right) \\
0 \\
0 \\
-(b-1) b^{3}\left(d^{2}-2 d+1\right) D^{3}\left(D_{\mathrm{N}}^{2}-2 D_{\mathrm{N}}+1\right)
\end{array}\right)\right) .
$$

The vector spanning the null space-in this case, it is only one- has all zero entries only for the elements corresponding to the two guessing parameters $g_{1}$ and $g_{2}$. So only those two parameters are locally identified. Another unidentified submodel of model 2HTSM results from the imposition of equality constraints on the guessing and bias parameters: $g_{1}=a_{1}$ and $g_{2}=a_{2}$. Because submodels of the 2HTSM are not locally identified, the 2HTSM is only conditionally full rank.

Low-threshold models. In the 2HTSM, the two high detection thresholds ensure that new items are never detected as old, and old items are never detected as new. Allowing new items to be — erroneously—detected as old results in the following unidentified low-threshold model

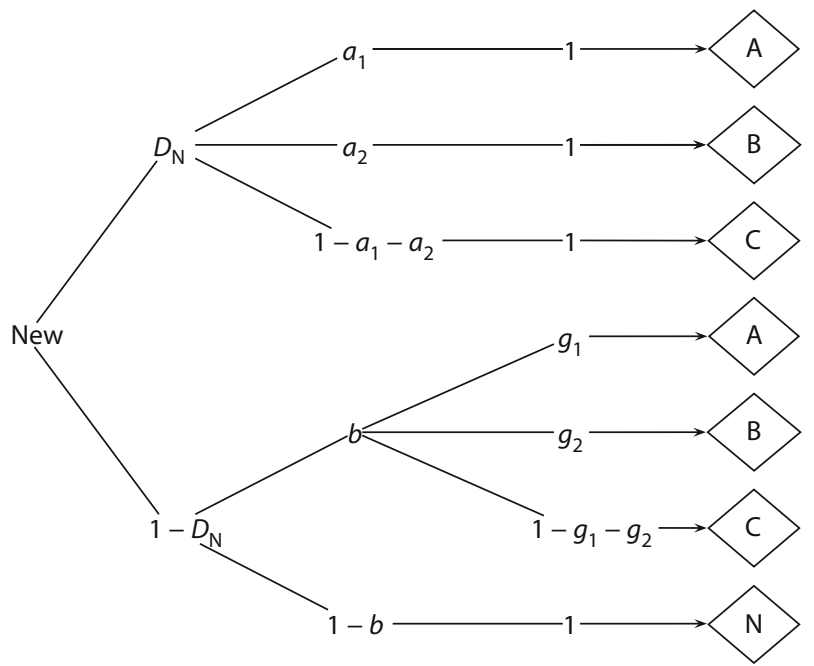

Figure 3. Processing tree of new items in the low-threshold MPT model of source monitoring for three sources. $D_{\mathrm{N}}=$ probability of detecting new items as old; $a_{1}\left(a_{2}, 1-a_{1}-a_{2}\right)=$ probability of guessing that a detected item comes from Source A $(B, C)$; $b=$ probability of guessing that an undetected item is old; $g_{1}\left(g_{2}\right.$, $\left.1-g_{1}-g_{2}\right)=$ probability of guessing that an undetected item comes from Source $A(B, C)$, given that it was guessed as being old. NB: The trees for $\mathrm{A}, \mathrm{B}, \mathrm{C}$ source items are equal to those in Figure 2.

(LTSM; Batchelder, Hu, \& Riefer, 1994). Old items are processed exactly as in the 2HTSM (see Figure 2). The amended processing tree for new items is shown in Figure 3. New items are erroneously detected as old with probability $D_{\mathrm{N}}$, and then classified as Source A, B, or C, with probabilities $a_{1}, a_{2}$, and $1-a_{1}-a_{2}$, respectively. Undetected new items are classified as A, B, C, or N, as in the 2HTSM above.

This version of an LTSM is not identified. Batchelder, Riefer, and $\mathrm{Hu}$ (1994) proposed to fix both the bias $b$ for the old items and the detection parameter of the new items $D_{\mathrm{N}}$ to zero to arrive at a theoretically relevant and identified submodel LTSM* ${ }^{*}$. Model LTSM* has received criticism and provided an inferior fit to source-monitoring data as compared with the 2HTSM (Bayen et al., 1996). However, there are additional ways of arriving at an identified model. Figure 4 shows a nested hierarchy of other submodels of interest, and whether or not those are locally identified by the Jacobian rank test. The submodels 11a, 10a, 11e, and $10 \mathrm{e}$ are locally identified, and may resolve misfit of the LTSM$^{*}$ version (Bayen et al., 1996). Note that these models are conditionally identified, because their respective submodels $8 \mathrm{ab}$ and $8 \mathrm{ce}$ are parameter redundant.

\section{Other Parameterizations}

In the above, we have parameterized the model by exploiting the fact that the probabilities associated with branchings sum to one. For instance, we parameterized a bifurcation by considering just one parameter (e.g., $b$ and $1-b)$, and in a trifurcation we considered two parameters (e.g., $a_{1}, a_{2}$, and $1-a_{1}-a_{2}$ ). This parameterization simplifies the model specification and reduces the col- 


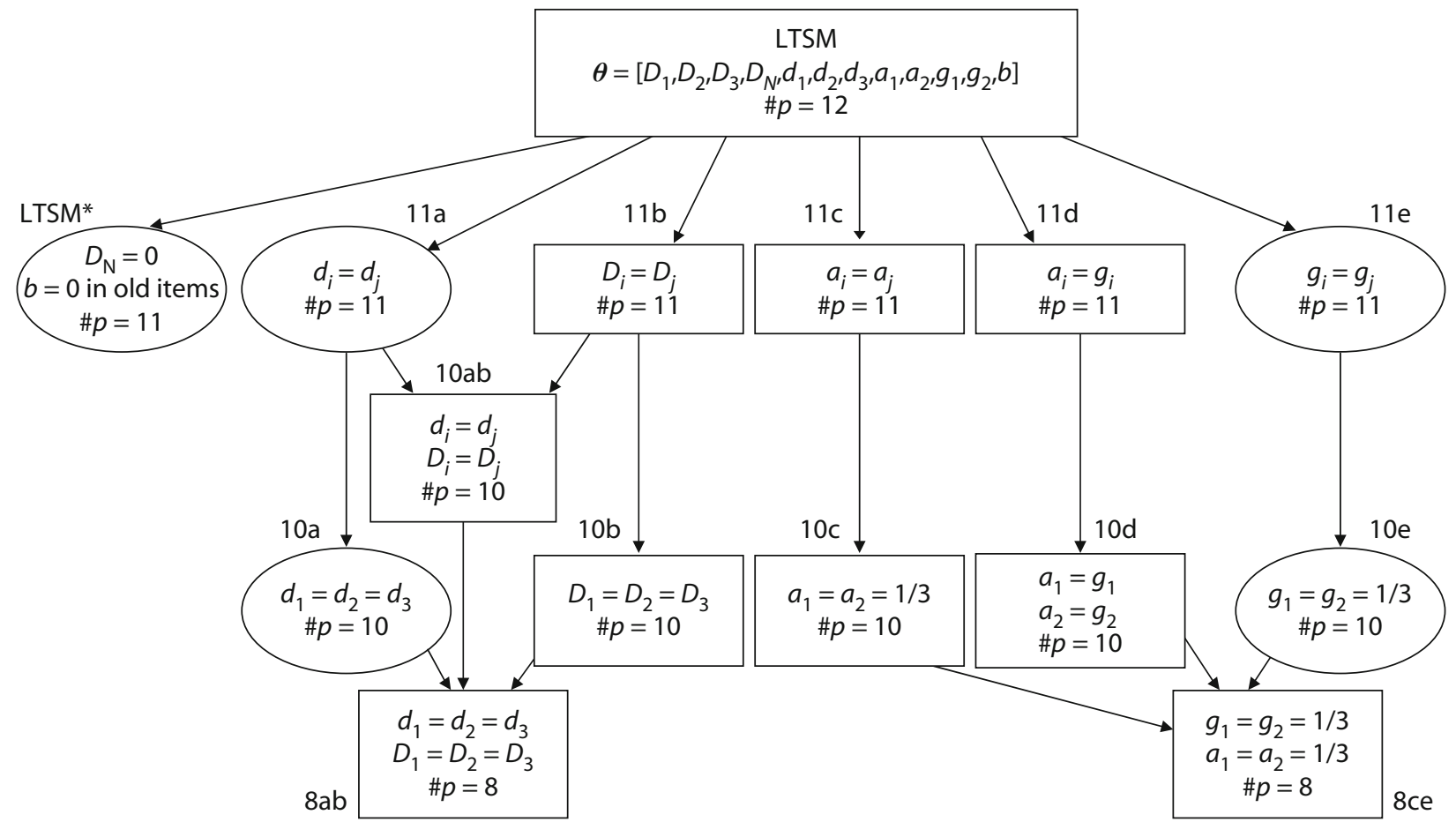

Figure 4. A nested hierarchy of submodels of interest of the low-threshold model (LTSM) with free parameters $\theta^{\mathrm{t}}=\left[D_{1}, D_{2}, D_{3}\right.$, $\left.D_{\mathrm{N}}, d_{1}, d_{2}, d_{3}, a_{1}, a_{2}, g_{1}, g_{2}, b\right]$. Arrows point from the respective supermodel to its nested submodels. Parameter constraints and the number of free parameters ( $\# p)$ of the models are indicated. Models in ovals are locally identified; models in rectangles are parameter redundant.

umn dimension of the Jacobian matrix. In actually fitting the model, this parameterization may run into difficulties in the case of a trifurcation; specifically, given the parameterization $a_{1}, a_{2}$, and $a_{3}=1-a_{1}-a_{2}$, boundary constraints $0<a_{1}<1$ and $0<a_{2}<1$ do not necessarily ensure that $0<a_{3}<1$. To avoid this problem in actually fitting the model, it may be more convenient to formulate the optimization problem using explicit linear constraints. For instance, one can estimate $a_{1}, a_{2}$, and $a_{3}$ as free parameters subject to the boundary constraint $\left(0<a_{i}\right.$, $i=1,2,3)$ and the linear constraint $1=\sum_{i=1}^{3} a_{i}$. We point out that with this parameterization, local identification can still be investigated by considering the column rank of the Jacobian. The dimension of vector $\boldsymbol{f}(\boldsymbol{\theta})$ is increased by adding the linear constraint (e.g., $a_{1}+a_{2}+a_{3}=1$ ), and the column dimension of the Jacobian is increased by the number of additional parameters (e.g., $a_{3}$ ). We emphasize that this parameterization results in the same conclusions concerning the rank of the Jacobian and the assessment of local identification. Maxima and Maple input files employing this parameterization for the illustrations above are available on request. The calculation of the expected information matrix remains relatively simple, but does include the Jacobian of the identifying constraints (e.g., $\left.1=\sum_{i=1}^{3} a_{i}\right)$. We refer the reader to Aitchison and Silvey (1958) for the relevant details.

\section{Discussion}

The analytical solution to the problem whether a given MPT model is globally identified may be intractable, even using programs like Maple and Maxima. One may then resort to establishing local identification. This can be done numerically or, as we have discussed here, analytically, by inspecting the rank of the Jacobian matrix. Advantages of the analytic approach are that it is not necessary to choose arbitrary parameter values, that data simulations can be avoided, and that it is possible to use any parameterization of the model. The specification of the MPT model and the required symbolic manipulations pose no problem for Maple and Maxima. In case the model is not identified, the output of Maxima and Maple includes specific information concerning which parameters in the model are not identified. This is useful, because it suggests which constraints will render the model locally identified. With a variety of identifying constraints at one's disposal, one can determine which identifying constraints (if any) are theoretically defensible.

By the imposition of equality constraints, a nested model can be derived from a locally identified model. Even though one is estimating a smaller number of parameters in the nested model, the constraints may render a conditionally identified model unidentified. It is, therefore, important that local identification be checked for all nested models (see Figure 4). As mentioned, identifying constraints in the nested model can be derived from the Maple or Maxima output. Furthermore, the imposition of appropriate boundary, inequality, or order constraints in a locally identified model may yield a globally identified model.

In this article, we have focused on establishing local identification in MPT models. It should be noted that 
both locally and globally identified models may run into computational difficulties due to empirical underidentification. This may happen when the parameters assume values close to a point or a region in the parameter space where the model is not identified - that is, the boundaries, eventual identifying constraints, or close to the parameter values of a locally unidentified submodel (Catchpole et al., 2001). The closer the parameter values are to unidentified points, and the less data is available, the more severe the problem of empirical underidentification will be, ranging from near redundancy (sometimes denoted as weak identification) to an empirically redundant model. In the former case, the observed information matrix is near singular (ill-conditioned), whereas in the latter case, this matrix is singular. Specifically, one or more eigenvalues of the information matrix will approach or equal zero (Catchpole et al., 2001; Gimenez, Viallefont, Catchpole, Choquet, \& Morgan, 2004; Viallefont et al., 1998). Empirical underidentificationfor example, stemming from near-boundary values of one or more parameters early in the branching of an MPT model - may render parameters deeper in the tree structure in turn empirically underidentified. For instance, if parameter $b$ in Figure 2 is sufficiently close to zero, parameters $g_{1}$ and $g_{2}$ will suffer from empirical underidentification, too. Happily, the HMMTree software of Stahl and Klauer (2007) and the multiTree software of Moshagen (2010) calculate the information matrix. Empirical underidentification can be diagnosed by inspecting its eigenvalues as discussed above.

Although other-mostly a posteriori-methods are available to detect parameter redundancy and empirical underidentification (i.e., profile likelihood, inspection of the information matrix, or simulation methods), the present formal approach has the advantage of distinguishing between parameter redundancy and empirical underidentification and of distinguishing between identified and redundant parameters within a parameter-redundant model (Gimenez et al., 2004). The present method does not provide an easy way to distinguish between conditionally and essentially full rank models. The existence of an unidentified submodel proves that a model is conditionally full rank. Gimenez, Choquet, and Lebreton (2003) presented a way of distinguishing between essentially and conditionally full rank models by decomposing the transpose of the Jacobian matrix, which was further elaborated by Cole and Morgan (2007, 2008). This approach may be elaborated for MPT models.

We have considered the problem of establishing local identification in standard MPT models (Batchelder \& Riefer, 1999). Klauer (2006) and Stahl and Klauer (2007) proposed latent class hierarchical MPT models - that is, finite mixtures of MPT models. As finite mixture models, they are in general not globally identified, since the order of the components in the mixture is arbitrary. This is not generally a problem, but can be solved by the judicious imposition of inequality constraints. A necessary constraint for local identification is that the component MPT models are distinct; that is, at least one parameter has to differ over the components. Of course, the greater the number of distinct parameters, and the greater their numerical difference, the better the component separation. Poor component separation may result in empirical underidentification. A formal approach to establishing local identification is to treat the $M$-component mixture MPT as an $M$-independent group MPT model. A necessary condition for local identification of the mixture is that the Jacobian of the $M$-independent group model be full rank, and that the MPT models include at least one parameter that differs over the groups. Generally, the problem of empirical underidentification is more acute in mixture MPT models.

As mentioned, a nested submodel derived by the imposition of equality constraints from its locally identified nesting model is not necessarily locally identified; it is important, therefore, to consider the identification of both. Note that if the identification of both models has been established, one can readily calculate the power to reject the constraints associated with the nested model. Specifically, given (1) a chosen alpha, (2) a choice of parameters under the nesting model, and (3) sample size, one can fit the nested model to the expected (exact) summary statistics, generated by the nesting model. This yields a likelihood ratio statistic that asymptotically equals the noncentrality parameter of the noncentral chisquare distribution - that is, the distribution of the likelihood ratio of the nested model versus the nesting model. With this information and the degrees of freedom (difference in number of parameters of the nested and nesting model), one can readily calculate the power to reject the constraints. This method, quite simple to implement, has been discussed extensively in structural equation modeling (see, e.g., Saris \& Satorra, 1993; Satorra \& Saris, 1985). In the context of MPT models, this method has been outlined by Erdfelder, Faul, and Buchner (2005) and may easily be performed using the program multiTree (Moshagen, 2010). In the case of MPT models, the likelihood ratio test is subject to the standard regularity conditions. Of course, if one does not have a clear idea about the effect size, one can vary the parameter values to get a sense of the kind of model violation one is likely to detect with a given sample size.

\section{AUTHOR NOTE}

The authors thank Lourens Waldorp for his useful comments. V.D.S. acknowledges a travel grant from the Netherlands Organization for Scientific Research (NWO). The work of M.E.J.R. was funded by a VIDI grant of the NWO. W.H.B.'s work on this article was supported in part by a National Science Foundation grant to X. Hu and W.H.B. (Co-PIs, SES-0616657) and by a grant from the U.S. Air Force Office of Scientific Research (FA9550 -09-05). Correspondence concerning this article should be addressed to V. D. Schmittmann, Department of Psychological Methods, University of Amsterdam, Roetersstraat 15, Room A.514, 1018WB Amsterdam, The Netherlands (e-mail: v.d.schmittmann@uva.nl).

\section{REFERENCES}

Aitchison, J., \& Silvey, S. D. (1958). Maximum-likelihood estimation of parameters subject to restraints. Annals of Mathematical Statistics, 29, 813-828. doi:10.1214/aoms/1177706538

ANDERSEN, E. B. (1980). Discrete statistical models with social science applications. Amsterdam: North-Holland. 
Azzalini, A. (1996). Statistical inference based on the likelihood. London: Chapman \& Hall.

BASILEVSKY, A. (1983). Applied matrix algebra in the statistical sciences. Amsterdam: Elsevier.

Batchelder, W. H., Hu, X., \& Riefer, D. M. (1994). Analysis of a model for source monitoring. In G. H. Fischer \& D. Laming (Eds.), Contributions to mathematical psychology, psychometrics, and methodology (pp. 51-65). New York: Springer.

BATCHELDER, W. H., \& RiefER, D. M. (1990). Multinomial processing models of source monitoring. Psychological Review, 97, 548-564. doi:10.1037/0033-295X.97.4.548

Batchelder, W. H., \& Riefer, D. M. (1999). Theoretical and empirical review of multinomial process tree modeling. Psychonomic Bulletin \& Review, 6, 57-86.

BATCHELDER, W. H., \& RiefER, D. M. (2007). Using multinomial processing tree models to measure cognitive deficits in clinical populations. In R. W. J. Neufeld (Ed.), Advances in clinical cognitive science: Formal modeling of processes and symptoms (pp. 19-50). Washington, DC: American Psychological Association.

Batchelder, W. H., Riefer, D. M., \& Hu, X. (1994). Measuring memory factors in source monitoring: Reply to Kinchla. Psychological Review, 101, 172-176. doi:10.1037/0033-295X.101.1.172

Bayen, U. J., Murnane, K., \& Erdfelder, E. (1996). Source discrimination, item detection, and multinomial models of source monitoring. Journal of Experimental Psychology: Learning, Memory, \& Cognition, 22, 197-215. doi:10.1037/0278-7393.22.1.197

BekKer, P. A., Merckens, A., \& Wansbeek, T. J. (1994). Identification, equivalent models, and computer algebra. Boston: Academic Press.

Bishop, Y. M. M., Fienberg, S. E., \& Holland, P. W. (1975). Discrete multivariate analysis: Theory and practice. Cambridge, MA: MIT Press.

Bollen, K. A. (1989). Structural equations with latent variables. New York: Wiley.

Catchpole, E. A., Kgosi, P. M., \& Morgan, B. J. T. (2001). On the near-singularity of models for animal recovery data. Biometrics, $\mathbf{5 7}$, 720-726. doi:10.1111/j.0006-341X.2001.00720.x

Catchpole, E. A., \& Morgan, B. J. T. (1997). Detecting parameter redundancy. Biometrika, 84, 187-196. doi:10.1093/biomet/84.1.187

Catchpole, E. A., \& Morgan, B. J. T. (2001). Deficiency of parameter-redundant models. Biometrika, 88, 593-598. doi:10.1093/ biomet/88.2.593

Catchpole, E. A., Morgan, B. J. T., \& Freeman, S. N. (1998). Estimation in parameter-redundant models. Biometrika, 85, 462-468. doi:10.1093/biomet $/ 85.2 .462$

Catchpole, E. A., Morgan, B. J. T., \& Viallefont, A. (2002). Solving problems in parameter redundancy using computer algebra. Journal of Applied Statistics, 29, 625-636. doi:10.1080/02664760120108601

Cole, D. J., \& Morgan, B. J. T. (2007). Detecting parameter redundancy in covariate models (University of Kent Tech. Rep. No. UKC/ IMS/07/007). Retrieved July 15, 2010, from www.kent.ac.uk/ims/ personal/djc24/TRColeandMorgan2007.pdf.

Cole, D. J., \& Morgan, B. J. T. (2008). Parameter redundancy and identifiability (University of Kent Tech. Rep. No. UKC/IMS/08/022, IMSAS). Retrieved July 15, 2010, from www.kent.ac.uk/ims/personal/ djc24/paramterredundancy.pdf.

DoDIER, R. (2009). Maxima, a computer algebra system (Version 5.20.1) [Computer software manual]. Retrieved from http://maxima .sourceforge.net/.

Dolan, C. V., \& Molenaar, P. C. M. (1991). A comparison of four methods of calculating standard errors of maximum likelihood estimates in the analysis of covariance structure. British Journal of Mathematical \& Statistical Psychology, 44, 359-368.

Erdfelder, E., Auer, T., Hilbig, B. E., Aßfalg, A., Moshagen, M., \& NADAREvic, L. (2009). Multinomial processing tree models: A review of the literature. Zeitschrift für Psychologie, 217, 108-124. doi:10.1027/0044-3409.217.3.108

Erdfelder, E., Faul, F., \& Buchner, A. (2005). Power analysis for categorical methods. In B. S. Everitt \& D. C. Howell (Eds.), Ency- clopedia of statistics in behavioral science (Vol. 3, pp. 1565-1570). Chichester, U.K.: Wiley.

Gimenez, O., Choquet, R., \& Lebreton, J.-D. (2003). Parameter redundancy in multistate capture-recapture models. Biometrical Journal, 45, 704-722. doi:10.1002/bimj.200390043

Gimenez, O., Viallefont, A., Catchpole, E. A., Choquet, R., \& MoRGAN, B. J. T. (2004). Methods for investigating parameter redundancy. Animal Biodiversity \& Conservation, 27, 561-572.

Hu, X., \& BATCHELDER, W. H. (1994). The statistical analysis of general processing tree models with the EM algorithm. Psychometrika, 59, 21-47. doi:10.1007/BF02294263

Hu, X., \& Phillips, G. A. (1999). GPT.EXE: A powerful tool for the visualization and analysis of general processing tree models. Behavior Research Methods, Instruments, \& Computers, 31, 220-234.

JöRESKOG, K. G., \& SöRBOM, D. (1989). LISREL 7: A guide to the program and applications. Chicago: Scientific Software International.

Keefe, R. S. E., Arnold, M. C., Bayen, U. J., McEvoy, J. P., \& WilSON, W. H. (2002). Source-monitoring deficits for self-generated stimuli in schizophrenia: Multinomial modeling of data from three sources. Schizophrenia Research, 57, 51-67. doi:10.1016/S0920 -9964(01)00306-1

KLAUER, K. C. (2006). Hierarchical multinomial processing tree models: A latent-class approach. Psychometrika, 71, 7-31. doi:10.1007/ s11336-004-1188-3

Meiser, T. (2005). A hierarchy of multinomial models for multidimensional source monitoring. Methodology, 1, 2-17. doi:10.1027/1614 $-1881.1 .1 .2$

MeISER, T., \& BRöDER, A. (2002). Memory for multidimensional source information. Journal of Experimental Psychology: Learning, Memory, \& Cognition, 28, 116-137. doi:10.1037/0278-7393.28.1.116

Monagan, M. B., Geddes, K. O., Heal, K. M., Labahn, G., Vorkoetter, S. M., McCarron, J., \& DeMarco, P. (2008). Maple 12 introductory programming guide [Computer software and manual]. Waterloo, ON: Maplesoft.

Moshagen, M. (2010). multiTree: A computer program for the analysis of multinomial processing tree models. Behavior Research Methods, 42, 42-54. doi:10.3758/BRM.42.1.42

READ, T. R. C., \& CRESSIE, N. A. C. (1988). Goodness-of-fit statistics for discrete multivariate data. New York: Springer.

Riefer, D. M., \& BATCHELDER, W. H. (1988). Multinomial modeling and the measurement of cognitive processes. Psychological Review, 95, 318-339. doi:10.1037/0033-295X.95.3.318

RothKegel, R. (1999). AppleTree: A multinomial processing tree modeling program for Macintosh computers. Behavior Research Methods, Instruments, \& Computers, 31, 696-700.

SARIS, W. E., \& SATORRA, A. (1993). Power evaluations in structural equation models. In K. A. Bollen \& J. S. Long (Eds.), Testing structural equation models (pp. 181-204). Newbury Park, CA: Sage.

Satorra, A., \& Saris, W. E. (1985). The power of the likelihood ratio test in covariance structure analysis. Psychometrika, 50, 83-90. doi:10.1007/BF02294150

Stahl, C. (2006). Multinomiale Verarbeitungsbaummodelle in der Sozialpsychologie [Multinomial processing tree models in social psychology]. Zeitschrift für Sozialpsychologie, 37, 161-171. doi:10.1024/0044-3514.37.3.161

Stahl, C., \& Klauer, K. C. (2007). HMMTree: A computer program for latent-class hierarchical multinomial processing tree models. $B e$ havior Research Methods, 39, 267-273.

Viallefont, A., Lebreton, J.-D., Reboulet, A.-M., \& Gory, G. (1998). Parameter identifiability and model selection in capturerecapture models: A numerical approach. Biometrical Journal, 40, 313-325. doi:10.1002/(SICI) 1521-4036(199807)40:3<313::AID -BIMJ313>3.0.CO;2-2

\section{NOTE}

1. Note that Bishop et al. (1975) expressed $\mathrm{I}(\boldsymbol{\theta})=A^{\mathrm{t}} A$, where $A=$ $F(\boldsymbol{\theta})^{1 / 2} \mathrm{~J}(\boldsymbol{\theta})$. This implies $A^{\mathrm{t}}=\mathrm{J}(\boldsymbol{\theta})^{\mathrm{t}} F(\boldsymbol{\theta})^{\mathrm{t} / 2}$, which gives $A^{\mathrm{t}} A=\mathrm{J}(\boldsymbol{\theta})^{\mathrm{t}} F(\boldsymbol{\theta}) \mathrm{J}(\boldsymbol{\theta})$. 


\section{APPENDIX}

Maxima Input Code for Three-Source 2HTSM

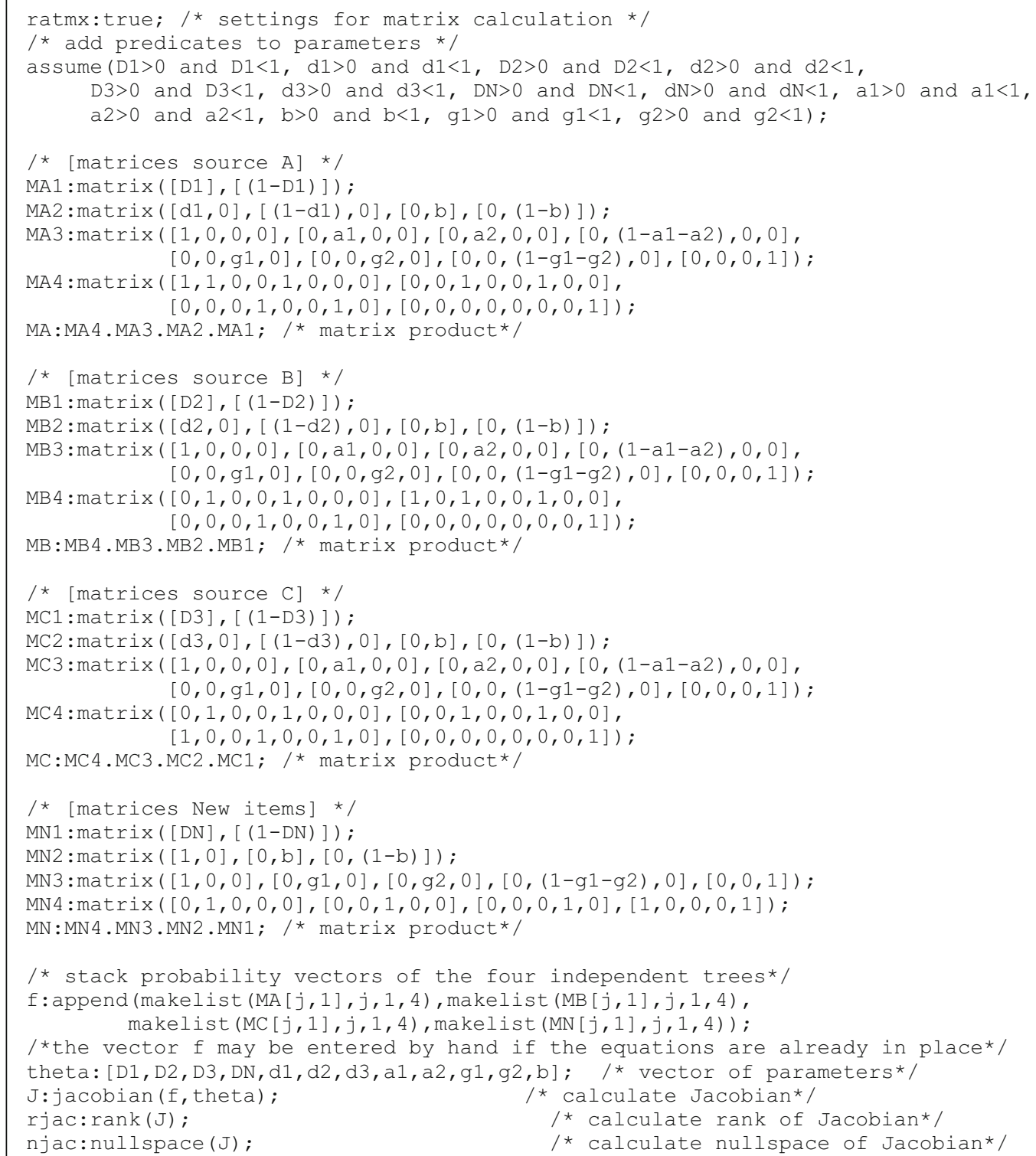

Maxima input 